\title{
Correspondence
}

\section{Acute arthritis and arthralgia as an adverse drug reaction to dupilumab}

\author{
doi: $10.1111 /$ ced.14050
}

Dupilumab is the first biologic registered for the treatment of atopic dermatitis (AD). We report a case of acute-onset monoarthritis of the ankle and generalized polyarthralgia in a 38-year-old woman with $\mathrm{AD}$, shortly after starting dupilumab. Methotrexate (MTX) therapy for AD had been discontinued 1 week before start of dupilumab treatment because of decreasing effectiveness. Four weeks after starting dupilumab, the patient reported improvement of her eczema; however, she also reported severe joint pain and morning stiffness in one ankle, which gradually spread to her knees, hips and elbows. These problems started a few days after the first dupilumab injection. She had not contacted us earlier because she was relieved by the rapid decrease in itch complaints. There was no history of previous joint pain and no family history of rheumatic diseases, and the patient's medical history was not contributory.

Rheumatological examination revealed tenderness of the wrists, knees and ankles. The right ankle was slightly swollen and warm. Laboratory investigations showed increases in inflammatory markers: C-reactive protein $33 \mathrm{mg} / \mathrm{L}$ (normal value $<10 \mathrm{mg} / \mathrm{L}$ ) and erythrocyte sedimentation rate $63 \mathrm{~mm} / \mathrm{h}(0-20 \mathrm{~mm} / \mathrm{h})$. Additional tests including serology screens for parvovirus B19, Epstein-Barr virus, Chlamydia trachomatis and Streptococcus were all negative.

Radiograph of the right ankle showed swollen tissue, without erosions or osteoarthritis.

Monoarthritis of the right ankle and inflammatory polyarthralgia as an adverse drug reaction to dupilumab were suspected, and dupilumab treatment was discontinued. The patient was started on a 1-month course of celecoxib $200 \mathrm{mg}$ twice daily and prednisolone $15 \mathrm{mg}$ daily for the joint pain, following which the arthritis and arthralgia disappeared. Six weeks after discontinuation of dupilumab, MTX (20 mg weekly, subcutaneously) was started because the patient's eczema flared. After
2 months, her eczema was controlled and the inflammatory markers had decreased.

The differential diagnosis included adverse drug reaction (ADR), serum sickness(-like reaction) [SS(LR)], peripheral spondyloarthropathy (SpA), and rheumatoid arthritis (RA). We considered ADR because of the clear timeline with onset of joint pain after first administration of dupilumab. ${ }^{1}$ To date, two cases of SS(LR) during dupilumab treatment have been reported. However, our patient denied the hallmark complaints of SS(LR), including fever, malaise and urticarial eruption, and the rapid onset of joint pain was not typical for SS(LR). ${ }^{2}$ RA and SpA were also considered. It has been suggested that patients with AD may have a higher risk of developing RA. ${ }^{3}$ In addition, dupilumab decreases levels of interleukin (IL)-4, which potentially can increase levels of tumour necrosis factor- $\alpha$ and IL-6, which are important inflammatory cytokines in the pathogenesis of RA. ${ }^{4}$ However, our patient did not have rheumatoid factor or anti-citrullinated protein antibodies, nor did she meet the RA or SpA criteria. ${ }^{5}$

As a result of the multiple drugs the patient received after discontinuation of dupilumab, it is difficult to assess whether the problems resolved spontaneously or as a result of the drugs that were administered. Furthermore, the previous use of MTX could also have masked the presence of possible pre-existing arthralgia. To our knowledge, this ADR has not been reported previously, and we hope this report will raise awareness of the potential for arthritis and arthralgia during dupilumab treatment.

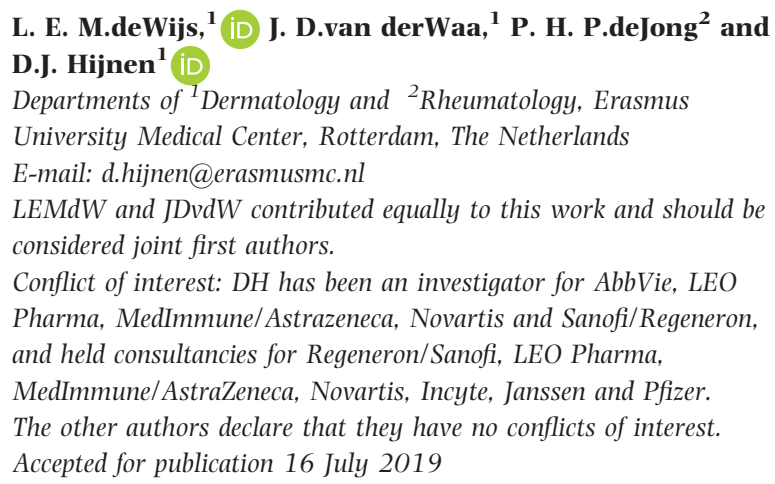




\section{References}

1 Mor A, Pillinger MH, Wortmann RL, Mitnick HJ. Druginduced arthritic and connective tissue disorders. Semin Arthritis Rheum 2008; 38: 249-64.

2 Frank MM, Hester CG. Immune complex-mediated diseases. In: Middleton's Allergy, 8th edn ( Adkinson NF, Bochner BS, Burks AW et al., eds). Philadelphia, PA: Elsevier Saunders, 2014; 602-16.

3 Schmitt J, Schwarz K, Baurecht H et al. Atopic dermatitis is associated with an increased risk for rheumatoid arthritis and inflammatory bowel disease, and a decreased risk for type 1 diabetes. J Allergy Clin Immunol 2016; 137: 130-6.

4 Dong $\mathrm{C}, \mathrm{Fu} \mathrm{T}$, Ji J et al. The role of interleukin- 4 in rheumatic diseases. Clin Exp Pharmacol Physiol 2018; 45: 747-54.

5 Aletaha D, Neogi T, Silman AJ et al. 2010 Rheumatoid arthritis classification criteria: an American College of Rheumatology/European League Against Rheumatism collaborative initiative. Ann Rheum Dis 2010; 69: 15808. 